\title{
Análise ontológica de definições de informação: em busca da sua essência
}

\author{
Ontological analysis of definitions of information: \\ In search for its essence
}

Carlos Henrique MARCONDES ${ }^{1}$

\section{Resumo}

As relações entre documento, informação e conhecimento não são nítidas e esses termos são frequentemente tomados de forma intercambiada. Quais as relações entre os conceitos informação, documento, conhecimento? Haveria algo que caracterizaria o ser de informação, sua essência, que o tornaria um fenômeno único, distinto? É proposta uma análise ontológica de diferentes definições de informação encontradas na literatura. Esta análise objetiva identificar semelhanças, diferenças, nuances ou variações. 0 trabalho tem como objetivo contribuir para uma teoria do documento e de suas relações com informação e conhecimento, que delimite informação como objeto próprio da Ciência da Informação.

Palavras-chave: Artefato. Conhecimento. Documento. Informação. Ontologia.

\begin{abstract}
The relation between documentation, information and knowledge are not clear and these terms are frequently used interchangeably. Which are the relations between the concepts of information, documentation and knowledge? Which would be the essence of information so as to precisely characterize it as a unique and distinct phenomenon? An ontological analysis of different definitions of information found in literature is proposed with the aim of identifying similarities, differences, nuances or variations. The aim of this study is to outline a theory of documentation and its relationship with information and knowledge in order to delimit information as an object of Information Science.
\end{abstract}

Keywords: Artifact. Knowledge. Document. Information. Ontology.

\section{Introdução}

Informação e/ou conhecimento são, de fato, "as duas faces de Jano". As relações entre documento, conhecimento e informação também não são nítidas, e esses termos são frequentemente tomados de forma intercambiada; a literatura da área registra recorrentemente o debate acerca dessas relações. Há bastante tempo a proliferação de acepções do termo é relatada de forma constante (Hjørland \& Albrechtsen, 1995). Informação é termo de extensão muito ampla, o que faz com que, na prática, seja empregado com múltiplos significados: "Information is notoriously a polymorphic phenomenon and a polimorphic concept so" (Floridi, 2013, online). Em (Højrland, 2012), são discutidos os conceitos de "information organization","organization of information", "information architecture" e"knowledge organization"; aí o termo informação é usado como um mero rótulo dado

\footnotetext{
1 Universidade Federal Fluminense, Instituto de Arte e Comunicação Social, Departamento de Ciência da Informação. R. Prof. Lara Vilela, 126, São Domingos, 24210-590, Niterói, RJ, Brasil. E-mail:<marcon@vm.uff.br>.

Recebido em 23/1/2014, reapresentado em 21/7/2014 e aceito para publicação em 25/8/2014.
} 
por comunidades de práticas, que não ajuda a distinguir o que, segundo o autor, são contextos de aplicação diferentes.

O termo também é usado na atualidade de forma coloquial, largamente empregado e frequentemente apropriado pela mídia e pelas empresas de consultoria, muitas vezes de forma metafórica; essa é uma das formas mais prejudiciais e deseducadoras de emprego de um termo para uma área científica. $O$ termo é replicado e reutilizado, e, o que é mais grave, muitas vezes pela própria Ciência da Informação (Cl), para designar entidades diferentes, em empregos que não explicitam seus pressupostos, aumentando a confusão conceitual. Outro emprego também tão comum quanto prejudicial a uma área científica, é atribuir à informação um sentido antropomorfo, como se informação tivesse algum tipo de intencionalidade, por exemplo "a informação causa... ou a informação faz com que...

No processo de busca de status científico, conforme relata a literatura que aborda aspectos históricos da Ciência da Informação (Burke, 2007), esta naturalmente se defrontou com a questão de estabelecer seus objetos, em especial, informação. Capurro e Hjørland (2003) arrolam as diferentes áreas que se interessam e conceituam informação, sua transversalidade e generalidade, as diferentes definições que existem dentro da Cl e os esforços desta para estabelecer seus "fundamental terms", entre os quais, a informação. Muitos autores, entre eles o próprio Burke (2007), afirmam que a inclusão da Cl no rol das Ciências Sociais desde a década de 1980 superou essa discussão, uma vez que a fundamentação teórica e conceitual das Ciências Sociais não é una, dividindo-se em visões, correntes ou escolas - tornando múltipla e polissêmica sua "terminologia de especialidade". Zins (2007, p.526), em artigo recente, afirma que "The eld of Information Science (IS) is constantly changing. Therefore, information scientists are required to regularly review, and if necessary, redene its fundamental building blocks". Essa afirmação juntamente com o recente verbete de Floridi (2013) na Stanford Enclyclopedia of Philosophy indicam o quanto o debate continua aberto e atual.

Assim, este trabalho endereça as seguintes questões: será que as diferentes acepções do termo informação não indicariam fenômenos diferentes, embora relacionados? Quais as relações e diferenças entre os con- ceitos informação, documento, conhecimento? Existiria ou não uma essência da informação, presente em todos esses fenômenos descritos pelas diferentes acepções do termo? O que caracterizaria o ser de informação, sua essência, que o tornaria um fenômeno único, distinto? Quais os pressupostos, muitas vezes não explícitos, de cada uma das acepções? São formas diferentes do mesmo fenômeno ou são fenômenos distintos? Informação seria uma entidade ou um processo?

O objetivo deste trabalho é, através de uma análise ontológica de definições de informação de autores seminais, identificar categorias e relações e explicitar e inventariar elementos envolvidos no fenômeno informação, contribuindo para uma teoria que englobe, relacione e diferencie documento, informação e conhecimento, e que delimite informação como objeto próprio da Cl. Nesse sentido, o trabalho objetiva contribuir para fundamentar a $\mathrm{Cl}$ ao propor identificar os objetos mencionados nos seus discursos e ao precisar seu significado, de modo a permitir que experiências possam ser comunicadas e comparadas.

A disciplina que pode contribuir para dar resposta às questões formuladas é a Ontologia. Smith e Welty (2001, p.3) afirmam: "Philosophical ontology is the science of what is, of the kinds and structures of objects, properties, events, processes and relations in every area of reality". Guarino (1995, p.628) destaca a utilização da análise ontológica na identificação de diferenças: "[...] formal ontology can be intended as the theory of a priori distinctions: among the entities of the world (physical objects, events, regions, quantities of matter [...]; among the meta-level categories used to model the world (concepts, properties, qualities, states, roles, parts".

As raízes da Ontologia podem ser buscadas em Aristóteles (2000), que fixou as bases de uma ciência do ser em obras como Categorias e Metafísica. Ontologia vem sendo retomada recentemente em função da necessidade crescente de se fixarem formalmente significados em um domínio de conhecimento para possibilitar o processamento desses significados, associados a recursos na Web, por computadores (Jacob, 2003). Nesse sentido, Ontologia é uma das áreas de pesquisa mais intensas para viabilizar a proposta da Web Semântica (Berners-Lee et al., 2001), que propiciará aplicações computacionais mais sofisticadas que farão uso intensivo do 
conhecimento, formalizado e registrado em novos artefatos: as ontologias computacionais.

Segundo Le Moigne (1990, p.79), uma completa compreensão de um fenômeno se dá segundo três dimensões: "A definição de um objeto faz-se por triangulação: pondera uma definição funcional (o que o objecto faz), uma definição ontológica (o que o objecto é) e uma definição genética (o que o objecto devém)". Este trabalho está assim organizado: após esta Introdução, seguem-se a seção que expõe a metodologia; a seção seguinte apresenta a análise das definições; a próxima seção traz a discussão dos resultados, e a última seção apresenta as conclusões.

\section{Procedimentos metodológicos}

A coordenação de ações humanas propiciada pela comunicação intersubjetiva através da linguagem (Knight et al., 2000) tornou-se um diferencial evolutivo decisivo na trajetória da humanidade. No entanto, de forma inerente, a comunicação via linguagem necessariamente caminha paripasso com um processo social contínuo de interpretação, ajuste dos significados e correção de imprecisões, que Brookes (1980b, p.209) chama de "objetivização".

A que entidades da realidade uma expressão linguística, seja ela uma elocução em linguagem natural, seja um texto, faz referência? Essa questão é endereçada pela Semântica, um dos três componentes ${ }^{2}$ da Semiótica (Morris, 1976), a ciência das linguagens como sistemas sociais e históricos de produção de sentido (Santaella, 1990). No caso da linguagem humana, essa correspondência foi e continua sendo fundamental para que a linguagem seja um instrumento de comunicação e articulação social; essa sua função se tornou um diferencial evolutivo para a espécie humana, como fundamento da Cultura (Marcondes, 2010). Por sua vez, a ciência que estuda quais entidades da realidade existem é a Ontologia, endereçando questões como:

It seeks not explanation but rather a description of reality in terms of a classification of entities that is exhaustive in the sense that it can serve as an answer to such questions as: What classes of entities are needed for a complete description and explanation of all the goings-on in the universe? Or: What classes of entities are needed to give an account of what makes true all truths? Or: What classes of entities are needed to facilitate the making of predictions about the future? (Smith, 2006, online).

O desenvolvimento de ontologias computacionais tem como base a Ontologia Formal, entendida como a ciência que lida com:"formal distinctions between the elements of a domain, independently of their actual reality" (Guarino, 1997, p.1); esse autor acrescenta ainda que "As such, formal ontology is a recent expression of traditional ontology, intended as the branch of philosophy which deals with the a priori nature of reality" (Guarino, 1997, p.2). Surgem várias propostas de ontologias de fundamentação que têm como base as ferramentas analíticas fornecidas pela Ontologia Formal e que servem como base e suporte para o desenvolvimento de ontologias computacionais em domínios específicos, propondo um repertório de categorias supremas, capazes de dar conta de representar qualquer domínio da realidade, como classe e subclasse, todo e parte, objetos e processos etc. Algumas dessas ontologias de fundamentação são Basic Formal Ontology (BFO) < http:// www.ifomis.org/bfo>, General Formal Ontology (GFO) $<$ http://www.onto-med.de/ontologies/gfo/>, Unified Foudational Ontology (UFO) <https://oxygen.informatik. tu-cottbus.de/drupal7/ufo/>, Foundational Model of Anatomy <http://sig.biostr.washington.edu/projects/fm/>

Uma dessas ontologias de fundamentação, usada nesta proposta, chama-se Descriptive Ontology for Linguistic and Cognitive Engineering (DOLCE) (Masolo et al., 2003). DOLCE tem seus elementos definidos formalmente através da lógica. Essas definições não são apresentadas aqui, pois fugiriam aos objetivos do trabalho, mas podem ser encontradas na referência citada.

Mesmo que seja recorrente na Filosofia a possibilidade de que a análise ontológica possa chegar à determinação última da natureza da realidade e que esta discussão seria antes uma discussão epistemológica do que uma discussão ontológica ${ }^{3}$, o uso de uma ontologia

\footnotetext{
2 Os outros são a Sintaxe, que estuda as sequências de elementos do vocabulário da linguagem aceitas como corretas, e a Pragmática, que estuda os usos sociais de uma linguagem.

3 Ver a discussão as diferentes posições com relação à natureza dos universais - realismo, nominalismo, conceitualismo -, em MacLeod e Rubinstein (2005).
} 
de fundamentação como DOLCE permite, no mínimo, unificar o vocabulário de diferentes definições de informação e permitir sua comparação.

Definições de informação são analisadas para determinar a que entidades e relações os termos aí encontrados se referem. Considera-se que a análise ontológica das definições de informação possa trazer aportes interessantes para a compreensão de seus referentes e ajudar a elucidar a natureza última do fenômeno informação. Para se chegar a essas determinações, é desenvolvido um processo aproximativo no qual, primeiro, os termos das definições ( $1^{\text {a }}$ coluna dos Quadros 1 a 4) são previamente identificados a categorias gramaticais (2a coluna dos Quadros 1 a 4, com base no WordNet <http:// wordnet.princeton.edu/>, um banco de dados com conceitos/termos no idioma inglês e sua categorias gramaticais; em alguns casos, substantivos que indiquem ações como "seleção"na definição de Capurro (2003) são identificados a verbos, como "selecionar". A seguir, cada termo é identificado às categorias do Modelo Entidades-Relacionamentos (E-R) de Chen (1976) - (3a coluna dos Quadros 1 a 4). A relação entre as categorias do modelo E-R e sua expressão linguística - entidades são associadas a substantivos, atributos a adjetivos e relacionamentos a verbos -, é fundamental na metodologia empregada para mapear com um mínimo de ambiguidades definições em linguagem natural às categorias da DOLCE. Essa relação é amplamente reconhecida na literatura (Hartmann \& Link, 2007).

A seguir, a Teoria dos Níveis da Realidade (Poli, 2001) e a ontologia dos três mundos (Popper, 1978) são utilizados para identificar o nível da realidade ao qual pertenceria a entidade da realidade a qual cada termo se refere (4a coluna dos Quadros 1 a 4); finalmente os termos são identificados às categorias ou relações da ontologia DOLCE (5a coluna dos Quadros 1 a 4). As categorias DOLCE identificadas são sempre apresentadas juntamente com a categoria de nível superior a que pertencem, para deixar mais claro seu contexto, como se segue: Object -> Agentive Physical Object. Quando não se trata de uma categoria, mas, sim, de uma relação, isto é assinalado, explicitando entre que categorias se dá a relação, como se segue: Relação Parthood (entre: information, knowledge). Para cada definição é apresen- tado um quadro com esses resultados. Ao final, esses quadros são discutidos.

Ontologias utilizadas: a ontologia DOLCE foi concebida como uma ontologia de alto nível, que permite que categorias de domínios específicos, como no caso do domínio documento-informação-conhecimento, pudessem ser referenciados e ter seus significados definidos. Segundo seus criadores

DOLCE has a clear cognitive bias ... We do not commit to a strictly referentialist metaphysics related to the intrinsic nature of the world: rather, the categories we introduce here are thought of as cognitive artifacts ultimately depending on human perception, cultural imprints and social conventions (Masolo et al., 2003, p.8).

Apresenta também um viés linguístico, que permite analisar as diferentes definições de informação e referenciá-las nas categorias DOLCE. Apesar de ser concebida como uma ontologia de particulares, ou seja, de entidades que ocorrem em tempos e espaços definidos, e não de universais, como talvez fosse mais conveniente em se tratando de análise de definições, sua vocação para tratamento da linguagem, cultura e cognição a tornaram uma escolha óbvia.

O modelo E-R (Chen, 1976, p.10), um metamodelo ou ontologia de alto nível de abstração, classifica as coisas existentes em três categorias: entidades: "a "thing" which can be distinctly identified"; relacionamentos: "an association among entities"; e atributos de entidades ou de relacionamentos: "The information about an entity or a relationship is obtained by observation or measurement, and is expressed by a set of attribute-value pairs". Assim, nos quadros de análise das definições, a coluna"Categoria Entidade-Relacionamento" pode conter os seguintes valores: entidades, relacionamentos ou atributos.

ATeoria dos Níveis da Realidade, conforme exposta em Poli (2001), oferece mais um elemento de sustentação para a identificação das categorias ontológicas dos elementos das definições e um mecanismo a mais de aproximação à identificação das categorias DOLCE. Calcada nas formulações de Hartmann, sustenta que a realidade é formada por níveis de crescente complexidade, cada um dos quais têm categorias e relações próprias e é por sua vez subdividido em diferentes estratos. Os níveis básicos utilizados nesta análise seriam o nível 
Quadro 1. Definição de Belkin e Robertson (1976) e Definição de Brookes (1980a).

\begin{tabular}{|c|c|c|c|c|c|}
\hline \multicolumn{6}{|c|}{ Definição de Belkin e Robertson (1976) } \\
\hline Entidade & $\begin{array}{l}\text { Categoria } \\
\text { gramatical }\end{array}$ & $\begin{array}{l}\text { Categoria } \\
\text { Entidades-Relacionamentos }\end{array}$ & Nível & $\begin{array}{l}\text { Categoria/Relação } \\
\text { DOLCE }\end{array}$ & Comentários \\
\hline Capable & Adjetivo & & & & $\begin{array}{l}\text { Potencialidade, não ne- } \\
\text { cessidade; modalidade de } \\
\text { ação. }\end{array}$ \\
\hline $\begin{array}{l}\text { transformin, } \\
\text { changing }\end{array}$ & Verbo & Relacionamento & & Event $->$ Achievement & \\
\hline Purposely & Adjetivo & Atributo & & & $\begin{array}{l}\text { Um "Agentive Physical } \\
\text { Object" é dotado de inten- } \\
\text { cionalidade. }\end{array}$ \\
\hline Sender & Substantivo & Entidade & Físico & $\begin{array}{l}\text { Physical Object ->Agentive } \\
\text { Physical Object }\end{array}$ & \\
\hline Recipient & Substantivo & Entidade & Físico & $\begin{array}{l}\text { Physical Object } \\
\text {->Agentive Physical Object }\end{array}$ & \\
\hline $\begin{array}{l}\text { structure, } \\
\text { image-structure }\end{array}$ & & Entidade & Mental & $\begin{array}{l}\text { Non-physical Object -> } \\
\text { Mental Object }\end{array}$ & \\
\hline Text & Substantivo & Entidade & Social objetivado & $\begin{array}{l}\text { Physical Object }->\text { Non- } \\
\text { agentive Physical Object }\end{array}$ & \\
\hline Signs & Substantivo & Entidade & Social objetivado & $\begin{array}{l}\text { Physical Object -> Non- } \\
\text { agentive Physical Object }\end{array}$ & \\
\hline \multicolumn{6}{|c|}{ Definição de Bookes (1980a) } \\
\hline Entidade & $\begin{array}{l}\text { Categoria } \\
\text { gramatical }\end{array}$ & $\begin{array}{l}\text { Categoria } \\
\text { Entidades-Relacionamentos }\end{array}$ & Nível & $\begin{array}{l}\text { Categoria/Relação } \\
\text { DOLCE }\end{array}$ & Comentários \\
\hline Knowledge & Substantivo & Entidade & Mental & $\begin{array}{l}\text { Non-physical } \\
\text { Object ->Mental Object }\end{array}$ & \\
\hline Structure & Substantivo & Entidade & Mental & & \\
\hline $\begin{array}{l}\text { Concepts, } \\
\text { (substructure of) }\end{array}$ & Substantivo & Entidade & Mental & $\begin{array}{l}\text { Non-physical Object } \\
->\text { Mental Object }\end{array}$ & \\
\hline $\begin{array}{l}\text { Linked by their } \\
\text { Relations }\end{array}$ & Verbo & Relacionamento & & & \\
\hline Information & Substantivo & Entidade & Mental & $\begin{array}{l}\text { Non-physical Object } \\
->\text { Mental Object }\end{array}$ & \\
\hline (small) part of & Substantivo & Relacionamento & & $\begin{array}{l}\text { Relação Parthood (entre: } \\
\text { information, knowledge) }\end{array}$ & \\
\hline (such a) structure & Substantivo & Entidade & & & \\
\hline \multirow[t]{4}{*}{ changed to by } & Verbo & Relacionamento & & Event $->$ Achievement & \\
\hline & & & & $\begin{array}{l}\text { Relação Participation } \\
\text { (entre: changed by, } \\
\text { information, } t \text { ) }\end{array}$ & \\
\hline & & & & $\begin{array}{l}\text { Relação Participation } \\
\text { (entre: changed to, } \\
\text { knowledge, } t \text { ) }\end{array}$ & \\
\hline & & & & $\begin{array}{l}\text { Relação Participation } \\
\text { (entre: changed to, new } \\
\text { knowledge, } t \text { ) }\end{array}$ & \\
\hline Information & Substantivo & Entidade & Mental & $\begin{array}{l}\text { Non-physical Object } \\
->\text { Mental Object }\end{array}$ & $\begin{array}{l}\text { Para o autor, informação } \\
\text { é da mesma natureza que } \\
\text { conhecimento. }\end{array}$ \\
\hline
\end{tabular}

Fonte: Elaborado pelo autor (2014).

Nota: DOLCE: Descriptive Ontology for Linguistic and Cognitive Engineering. 
Quadro 2. Definição de Farradane (1980) e Definição de Buckland (1991).

\begin{tabular}{|c|c|c|c|c|c|}
\hline \multicolumn{6}{|c|}{ Definição de Farradane (1980) } \\
\hline Entidade & $\begin{array}{l}\text { Categoria } \\
\text { gramatical }\end{array}$ & $\begin{array}{l}\text { Categoria } \\
\text { Entidades-Relacionamentos }\end{array}$ & Nível & $\begin{array}{l}\text { Categoria/Relação } \\
\text { DOLCE }\end{array}$ & Comentários \\
\hline $\begin{array}{l}\text { 'Information' is } \\
\text { defined as }\end{array}$ & & & & & $\begin{array}{l}\text { É o que está sendo defi- } \\
\text { nido. }\end{array}$ \\
\hline $\begin{array}{l}\text { a physical } \\
\text { surrogate }\end{array}$ & Substantivo & Entidade & Social objetivado & $\begin{array}{l}\text { Physical Object } \\
->\text { Non-agentive Physical } \\
\text { Object }\end{array}$ & $\begin{array}{l}\text { Um "surrogate" é um obje- } \\
\text { to que substitui ou "repre- } \\
\text { senta", em determinado } \\
\text { contexto social, a outro. }\end{array}$ \\
\hline $\begin{array}{l}\text { of knowledge (e.g. } \\
\text { language) }\end{array}$ & Substantivo & Entidade & Mental & $\begin{array}{l}\text { Non-physical Object } \\
->\text { Mental Object }\end{array}$ & \\
\hline used for & & & & & \\
\hline Communication & Verbo & Relacionamento & & Event $->$ Achievement & \\
\hline \multicolumn{6}{|c|}{ Definição de Buckland (1991) } \\
\hline Entidade & $\begin{array}{c}\text { Categoria } \\
\text { gramatical }\end{array}$ & $\begin{array}{l}\text { Categoria } \\
\text { Entidades-Relacionamentos }\end{array}$ & Nível & $\begin{array}{l}\text { Categoria/Relação } \\
\text { DOLCE }\end{array}$ & Comentários \\
\hline $\begin{array}{l}\text { Information-as- } \\
\text { process }\end{array}$ & & & & Event $->$ Achievement & $\begin{array}{l}\text { É o que está sendo defi- } \\
\text { nido. }\end{array}$ \\
\hline when someone & Substantivo & Entidade & Material & $\begin{array}{l}\text { Physical Object ->Agentive } \\
\text { Physical Object }\end{array}$ & \\
\hline is informed & Verbo & Relacionamento & & Event ->Achievement & \\
\hline what they know & Substantivo & Entidade & Mental & $\begin{array}{l}\text { Non-physical Object } \\
->\text { Mental Object }\end{array}$ & \\
\hline Is changed & Verbo & & & Event ->Achievement & $\begin{array}{l}\text { A referência é ao proces- } \\
\text { so ("is informed" e ao re- } \\
\text { sultado ("what they know } \\
\text { is changed"), mas não são } \\
\text { mencionados nem o in- } \\
\text { sumo, nem o contexto, } \\
\text { etc. }\end{array}$ \\
\hline $\begin{array}{l}\text { (The act of) } \\
\text { informing }\end{array}$ & Verbo & & & Event $->$ Achievement & \\
\hline $\begin{array}{l}\text { Information-as } \\
\text { knowledge }\end{array}$ & & & Mental & $\begin{array}{l}\text { Non-physical Object } \\
->\text { Mental Object }\end{array}$ & $\begin{array}{l}\text { É o que está sendo defi- } \\
\text { nido. }\end{array}$ \\
\hline which is perceived & Substantivo & Entidade & Mental & $\begin{array}{l}\text { Non-physical Object } \\
->\text { Mental Object }\end{array}$ & \\
\hline $\begin{array}{l}\text { in information-as- } \\
\text { process }\end{array}$ & Verbo & & & Event $->$ Achievement & \\
\hline Knowledge & Substantivo & Entidade & Mental & $\begin{array}{l}\text { Non-physical Object } \\
->\text { Mental Object }\end{array}$ & \\
\hline communicated & Verbo & Relacionamento & & Event ->Achievement & \\
\hline $\begin{array}{l}\text { Information- } \\
\text { asthing }\end{array}$ & Substantivo & & Material & $\begin{array}{l}\text { Physical Object }->\text { Non- } \\
\text { agentive Physical Object }\end{array}$ & $\begin{array}{l}\text { É o que está sendo defi- } \\
\text { nido. }\end{array}$ \\
\hline Objects & Substantivo & & Material & $\begin{array}{l}\text { Physical Object ->Non- } \\
\text { agentive Physical Object }\end{array}$ & \\
\hline $\begin{array}{l}\text { Having the quality } \\
\text { of }\end{array}$ & Adjetivo & & & & \\
\hline imparting & Verbo & & & Event $->$ Achievement & \\
\hline Knowledge & Substantivo & & Mental & $\begin{array}{l}\text { Non-physical Object } \\
\rightarrow>\text { Mental Object }\end{array}$ & \\
\hline communication & $\begin{array}{l}\text { Verbo } \\
\text { (substantivado } \\
\text { comunicar) }\end{array}$ & & & Event $->$ Achievement & \\
\hline Information & Substantivo & & Mental & $\begin{array}{l}\text { Non-physical Object } \\
->\text { Mental Object }\end{array}$ & \\
\hline
\end{tabular}

110

Fonte: Elaborado pelo autor (2014).

Nota: DOLCE: Descriptive Ontology for Linguistic and Cognitive Engineering. 
Quadro 3. Definição de Bates (2005), Definição de Raber e Budd (2003) e Definição de Capurro (2003).

\begin{tabular}{|c|c|c|c|c|c|}
\hline \multicolumn{6}{|c|}{ Definição de Bates (2005) } \\
\hline Entidade & $\begin{array}{l}\text { Categoria } \\
\text { gramatical }\end{array}$ & $\begin{array}{l}\text { Categoria } \\
\text { Entidades-Relacionamentos }\end{array}$ & Nível & $\begin{array}{l}\text { Categoria/Relação } \\
\text { DOLCE }\end{array}$ & Comentários \\
\hline Pattern & Substantivo & Entidade & & & \\
\hline organization & Substantivo & Entidade & & Abstract Quality & \\
\hline Matter & Substantivo & Entidade & Material & $\begin{array}{l}\text { Physical Endurant } \\
->\text { Amount of Matter }\end{array}$ & \\
\hline Energy & Substantivo & Entidade & Material & & \\
\hline has been given & Verbo & Relacionamento & & Event $->$ Achievement & \\
\hline meaning (by) & Substantivo & Entidade & Mental & $\begin{array}{l}\text { Non-physical Object } \\
\rightarrow \text { Mental Object }\end{array}$ & \\
\hline (a) living being & Substantivo & Entidade & Material & $\begin{array}{l}\text { Physical Object } \\
\rightarrow \text { Agentive Physical Object }\end{array}$ & \\
\hline
\end{tabular}

Knowledge

information

integrated with

other contents of understanding
Relacionamento

Mental

É o que está sendo definido.

Segundo a definição prévia, seriam estruturas mentais semióticas, significados.

Mental

Significados

\begin{tabular}{|c|c|c|c|c|c|}
\hline \multicolumn{6}{|c|}{ Definição de Raber e Budd (2003) } \\
\hline Entidade & $\begin{array}{l}\text { Categoria } \\
\text { gramatical }\end{array}$ & $\begin{array}{l}\text { Categoria } \\
\text { Entidades-Relacionamentos }\end{array}$ & Nível & $\begin{array}{l}\text { Categoria/Relação } \\
\text { DOLCE }\end{array}$ & Comentários \\
\hline \multicolumn{6}{|l|}{ Information } \\
\hline $\begin{array}{l}\text { (is a kind of) } \\
\text { sign }\end{array}$ & Substantivo & Entidade & Mental & $\begin{array}{l}\text { Non-physical Object } \\
->\text { Mental Object }\end{array}$ & \\
\hline (that) unites & $\begin{array}{l}\text { Verbo } \\
\text { (unificar) }\end{array}$ & Relacionamento & & Event ->Achievement & \\
\hline Text & Substantivo & Entidade & Social objetivado & $\begin{array}{l}\text { Physical Object } \\
\text {->Non-agentive Physical } \\
\text { Object }\end{array}$ & \\
\hline Content & Substantivo & Entidade & Social & $\begin{array}{l}\text { Non-physical Object } \\
->\text { Mental Object }\end{array}$ & \\
\hline \multicolumn{6}{|c|}{ Definição de Capurro (2003) } \\
\hline Entidade & $\begin{array}{l}\text { Categoria } \\
\text { gramatical }\end{array}$ & $\begin{array}{l}\text { Categoria } \\
\text { Entidades-Relacionamentos }\end{array}$ & Nível & $\begin{array}{l}\text { Categoria/Relação } \\
\text { DOLCE }\end{array}$ & Comentários \\
\hline diferença & Adjetivo & Atributo & Mental & & \\
\hline mensagem & Substantivo & Entidade & Mental & $\begin{array}{l}\text { Physical Object } \\
\text {->Non-agentive Physical } \\
\text { Object }\end{array}$ & \\
\hline oferta (oferecer) & Verbo & Relacionamento & & Event ->Achievement & \\
\hline de sentido & Substantivo & Entidade & Mental & $\begin{array}{l}\text { Non-physical Object } \\
->\text { Mental Object }\end{array}$ & \\
\hline informação & Substantivo & Entidade & & $\begin{array}{l}\text { Non-physical Object } \\
->\text { Mental Object }\end{array}$ & \\
\hline seleção & $\begin{array}{l}\text { Verbo } \\
\text { (selecionar) }\end{array}$ & Relacionamento & & Event $->$ Achievement & \\
\hline de sentido & Substantivo & Entidade & & $\begin{array}{l}\text { Non-physical Object } \\
->\text { Mental Object }\end{array}$ & \\
\hline
\end{tabular}

Fonte: Elaborado pelo autor (2014).

Nota: DOLCE: Descriptive Ontology for Linguistic and Cognitive Engineering. 
Quadro 4. 1a Definição de Frohman (2004), 2a Definição de Frohman (2004), Definição de Hjørland e Albrechtsen (1995) e Definição de Floridi (2013).

\begin{tabular}{|c|c|c|c|c|c|}
\hline \multicolumn{6}{|c|}{ 1a Definição de Frohman (2004) } \\
\hline Entidade & $\begin{array}{l}\text { Categoria } \\
\text { gramatical }\end{array}$ & $\begin{array}{l}\text { Categoria } \\
\text { Entidades-Relacionamentos }\end{array}$ & Nível & $\begin{array}{l}\text { Categoria/Relação } \\
\text { DOLCE }\end{array}$ & Comentários \\
\hline $\begin{array}{l}\text { the key } \\
\text { properties of }\end{array}$ & & Atributo & & & \\
\hline $\begin{array}{l}\text { abstract } \\
\text { impression of }\end{array}$ & Substantivo & Entidade & Mental & $\begin{array}{l}\text { Non-physical Object } \\
->\text { Mental Object }\end{array}$ & \\
\hline $\begin{array}{l}\text { information-as- } \\
\text { substance }\end{array}$ & Substantivo & Entidade & Social & $\begin{array}{l}\text { Physical Endurant } \\
\text {->Amount of Matter }\end{array}$ & \\
\hline $\begin{array}{l}\text { are simply the } \\
\text { reifications of } \\
\text { (reificar) }\end{array}$ & Verbo & Relacionamento & & Event $->$ Achievement & \\
\hline $\begin{array}{l}\text { the various } \\
\text { principles of } \\
\text { interpretation } \\
\text { (interpreter) }\end{array}$ & Verbo & Relacionamento & & Event $->$ Achievement & \\
\hline We & Substantivo & Entidade & & $\begin{array}{l}\text { Physical Object ->Agentive } \\
\text { Physical Object }\end{array}$ & \\
\hline bring to reading & Verbo & Relacionamento & & Event $->$ Achievement & \\
\hline $\begin{array}{l}\text { (specific, } \\
\text { historically } \\
\text { contingent) } \\
\text { document (forms) }\end{array}$ & Substantivo & Relacionamento & Social objetivado & $\begin{array}{l}\text { Physical Object } \\
\text {->Non-agentive Physical } \\
\text { Object }\end{array}$ & \\
\hline
\end{tabular}

\begin{tabular}{|c|c|c|c|c|c|}
\hline \multicolumn{6}{|c|}{ 2a Definição de Frohman (2004) } \\
\hline Entidade & $\begin{array}{l}\text { Categoria } \\
\text { gramatical }\end{array}$ & $\begin{array}{l}\text { Categoria } \\
\text { Entidades-Relacionamentos }\end{array}$ & Nível & $\begin{array}{l}\text { Categoria/Relação } \\
\text { DOLCE }\end{array}$ & Comentários \\
\hline $\begin{array}{l}\text { Shift away (our } \\
\text { attention) }\end{array}$ & Verbo & Relacionamento & & Event $->$ Achievement & \\
\hline $\begin{array}{l}\text { (from) mentalistic } \\
\text { pictures of } \\
\text { information }\end{array}$ & Substantivo & Entidade & Social & $\begin{array}{l}\text { Non-physical Object } \\
->\text { Mental Object }\end{array}$ & \\
\hline $\begin{array}{l}\text { (toward) practices } \\
\text { (with) }\end{array}$ & Verbo & Relacionamento & & Event $->$ Achievement & \\
\hline Documents & Substantivo & Entidade & Social objetivado & $\begin{array}{l}\text { Physical Object } \\
\text {->Non-agentive Physical } \\
\text { Object }\end{array}$ & \\
\hline \multicolumn{6}{|c|}{ Definição de Hjørland e Albrechtsen (1995) } \\
\hline Entidade & $\begin{array}{l}\text { Categoria } \\
\text { gramatical }\end{array}$ & $\begin{array}{l}\text { Categoria } \\
\text { Entidades-Relacionamentos }\end{array}$ & Nível & $\begin{array}{l}\text { Categoria/Relação } \\
\text { DOLCE }\end{array}$ & Comentários \\
\hline $\begin{array}{l}\text { Understand } \\
\text { Information in IS }\end{array}$ & Verbo & Relacionamentos & & & \\
\hline to study & Verbo & Relacionamento & & & \\
\hline $\begin{array}{l}\text { knowledge } \\
\text { domain }\end{array}$ & Substantivo & Entidade & Social & $\begin{array}{l}\text { Social Object } \\
->\text { Non-agentive }\end{array}$ & \\
\hline Thought & Substantivo & Entidade & Social & $\begin{array}{l}\text { Social Object } \\
->\text { Non-agentive Social } \\
\text { Object }\end{array}$ & \\
\hline $\begin{array}{l}\text { discourse } \\
\text { communities }\end{array}$ & Substantivo & Entidade & Social & $\begin{array}{l}\text { Agentive Social Object } \\
->\text { Society }\end{array}$ & \\
\hline
\end{tabular}


Quadro 4. 1a Definição de Frohman (2004), 2a Definição de Frohman (2004), Definição de Hjørland e Albrechtsen (1995) e Definição de Floridi (2013).

2 de 2

\begin{tabular}{|c|c|c|c|c|c|}
\hline \multicolumn{6}{|c|}{ Definição de Floridi (2013) } \\
\hline Entidade & $\begin{array}{l}\text { Categoria } \\
\text { gramatical }\end{array}$ & $\begin{array}{l}\text { Categoria } \\
\text { Entidades-Relacionamentos }\end{array}$ & Nível & $\begin{array}{l}\text { Categoria/Relação } \\
\text { DOLCE }\end{array}$ & Comentários \\
\hline Data & Substantivo & Entidade & Material & Physical Object & \\
\hline Meaning & Substantivo & Entidade & Mental & $\begin{array}{l}\text { Non-physical Object } \\
->\text { Mental Object }\end{array}$ & \\
\hline
\end{tabular}

Fonte: Elaborado pelo autor (2014).

Nota: DOLCE: Descriptive Ontology for Linguistic and Cognitive Engineering.

material ou físico, o mental e o social. Segundo Gnoli (2011), os níveis propostos por Poli (2001) são equivalentes aos 3 mundos de Popper (1978). A utilização da ontologia de Popper combinada à de Poli é interessante para os fins deste trabalho, na medida que Popper dá ênfase, entre os objetos do seu mundo 3 (nível social, de Poli), àqueles corporificados ou objetivados "World 1 embodiments of world 3 object, such as handwritten books, or printed books... (Popper, 1978, p.162), os objetos da cultura e do conhecimento com que tradicionalmente lida a Cl. Nos quadros de análise das definições, a coluna "Nível" pode então conter os seguintes valores: material, mental, social e social objetivado (Poli, 2001), para os objetos da cultura e do conhecimento, os também chamados artefatos ou mundo 3 (Popper, 1978).

As ontologias utilizadas, ao oferecerem um quadro comparativo a partir de diferentes pontos de vista, ajudam a caracterizar mais precisamente a natureza última dos objetos determinados pelos termos das definições. Permitem também maior precisão na identificação da categoria DOLCE de cada um deles ao explicitarem etapas, constituídas por cada uma das ontologias usadas como intermediárias WordNet, Modelo E-R e Níveis da Realidade - do mapeamento de termos linguísticos das definições nas categorias da DOLCE.

Foram analisadas 11 definições de autores dos mais citados, entre as mais plurais e representativas de diferentes visões, algumas vezes chamadas de "paradigmas" da Ciência da Informação (Capurro, 2003). A maioria das definições utilizadas está em artigos citados neste texto. A falta de definições formais representativas do "paradigma físico" levou à inclusão de versões mais recentes representativas do mesmo paradigma (Farradane, 1980; Bates, 2005). O artigo de Frohmann (2004) foi incluído por trazer um questionamento à própria existência de um fenômeno informação. Por fim, o recente verbete de Floridi (2013), na Stanford Encyclopedia of Philosophy, publicação que transcende a área de $\mathrm{Cl}$, foi incluído dada sua atualidade. A maioria das definições consta de artigos que foram publicados em periódicos da área de $\mathrm{Cl}$.

Definições são um dos pilares de uma ciência. Dahlberg $(1981,1983)$, uma das autoras que mais trabalhou a definição e sua importância para a organização do conhecimento, afirma que estas são "pressupostos indispensáveis na argumentação e nas comunicações verbais e que constituem elementos necessários na construção de sistemas científicos" (Dahlberg, 1978, p.106). Manuais de metodologia científica destacam a importância da definição para a ciência. Segundo Viegas (2007, p.73):"A ciência também tem seus instrumentos - como a definição e a classificação - e exigências rituais - como a argumentação". Definição é uma frase que exprime o significado de algo. Segundo o mesmo autor, definição "é uma locução que exprime o que uma coisa é ou qual o significado de um termo. A definição busca atingir a essência do definido, aquilo que faz com que a coisa seja ela e não outra..." (Viegas, 2007, p.75). Essa conceituação deixa clara a ligação entre definição e Ontologia.

Numa definição, o termo a ser definido denomina-se definiendum. Uma definição formal procura praticamente igualar o definiendum com a definição, na forma A é B, ou A = B. Procurou-se extrair da literatura definições 
de informação que fossem dessa forma ou se aproximassem dela.

\section{Análise das definições}

Para Belkin e Robertson - Information is that which is capable of transforming structure (1976, p.198).

A Text (in information science) is a collection of signs purposely structured by a sender with the intention of changing the image-structure of a recipient. Information (in information science) is the structure of any text which is capable of changing the imagestructure of a recipient (Belkin \& Robertson, 1976, p.201).

O propósito de Belkin e Robertson (1976, p.197) é determinar "the fundamental phenomena of interest to information science". Os autores delimitam sua proposta: "information science is specifically concerned with information in the context of human communication" (Belkin \& Robertson, 1976, p.198).

Informação é definida a partir de uma função que envolve, de forma explícita, agentes, possivelmente humanos e dotados de intencionalidade (sender e recipient), estados mentais (structure, image-structure), processos sígnicos (transforming, changing) e objetos/artefatos (text, signs). Para os autores, "structure" e "image-structure" são "animal's conceptual view" (Belkin \& Robertson, 1976, p.198), a visão do mundo que animais superiores, em especial humanos, constroem a partir de estímulosexperiências na sua interação com o mundo. Essa visão conceitual poderia ser alterada de duas maneiras: a partir de novos estímulos-experiências ou através de "messages received from other humans (and structured by them)" (Belkin \& Robertson, 1976, p.199) de forma intencional ("purposely"). Essa visão de informação integra o nível 114 mental ao social objetivado.

What is the relation between information and knowledge? I regard knowledge as a structure of concepts linked by their relations and information as a small part of such a structure.

[...] l expressed this relationship by what I called the 'fundamental equation': $\mathrm{K}[\mathrm{S}]+\Delta \mathrm{K}=\mathrm{K}[\mathrm{S}+\Delta \mathrm{S}]$, Which states in its very general way that the knowledge structure $\mathrm{K}[\mathrm{S}]$ is changed to the new modified structure $\mathrm{K}[\mathrm{S}+\Delta \mathrm{S}]$ by the information $\Delta \mathrm{l}$, the $\Delta \mathrm{S}$ indicating the effect of the modification (Brookes, 1980a, p.131).
A definição de Brookes (1980a) traz dois verbos: "linked by" e "changed by" e três relações: os dois verbos anteriores, mais a relação "part of'. A relação "linked by" é entre "concepts" e suas "relations". A relação "part of" aparece duas vezes: a primeira, entre"concepts"e "knowledge", e a segunda, entre "information" e "knowledge". Ambos, "information" e "knowledge", participam da relação "changed by", a relação realmente significativa. As relações da DOLCE são somente binárias; assim, tanto "information" quando"knowledge" estão envolvidas, através de relações DOLCE "participation", com "changed by", cuja categoria DOLCE é evento ("Event").

Resta examinar possíveis pressupostos da definição de Brookes (1980a). Todas as entidades na definição de Brookes (1980a) são objetos puramente mentais. A única exceção a um processo unicamente mental é a entidade "concepts". Na concepção da Semiótica Peirceana, um conceito é parte da relação, dita triádica, do signo, que envolve ainda um objeto a que o signo se refere; o conceito é chamado de interpretante, a representação mental do objeto para um intérprete. Segundo Peirce (1977, p.46): "o signo é qualquer coisa que é, de um lado, de tal modo é determinado por um objeto e, por outro lado, de tal modo determina uma ideia na mente de alguém que essa última determinação, chamada de interpretante do signo, é consequentemente determinada mediatamente por aquele objeto". Um agente humano, responsável pelos processos que envolvem esses objetos mentais, seria um pressuposto da definição de Brookes (1980a). Já Dahlberg (1995), apesar de reconhecer no conceito uma unidade de conhecimento, o conceitua de forma objetiva, como uma manifestação linguística dessa unidade de conhecimento, o termo, que se refere a um objeto do mundo e que por sua vez sintetiza seu significado.

Mais adiante, Brookes (1980a, p.131) afirma que: "Information may, of course, depend on sensory observation, but the sense-data so received have to be subjectively interpreted by a knowledge structure to became information". Interpretação é um processo semiótico que pressupõe um ator humano que, com base no seu conhecimento ("knowledge structure"), possa interpretar dados e, segundo Brookes (1980a), transformá-los em informação. Assim, dados seriam interpretados (por alguém), tendo como insumo (estruturas de) conhecimento, e como produto, informação. 
Para Farradane (1980, p.77) "[...] knowledge is defined as a memorable Record of a process in the brain; the production of consciousness in the mind is at present quite unexplained. 'Information' is defined as a physical surrogate of knowledge (e.g. language) used for communication".

Na visão do autor, as experiências vividas resultariam em registros destas mesmas experiências na mente, recuperáveis ("memorable Record") e constituindo o conhecimento; informação seriam objetos físicos que representam esse conhecimento ("surrogate") através da linguagem, usados para comunicação, isto é, documentos. Essa visão de informação integra processos mentais - conhecimento - a objetos físicos socialmente instituídos com essa finalidade.

1. Information-as-process: when someone is informed, what they know is changed. [...] The act of informing [...]

2. Information-as knowledge: Information is also used to denote which is perceived in informationas-process: the knowledge communicated concerning some particular fact, subject, or event.

3. Information-as-thing: the term information is also attributively for objects... having the quality of imparting knowledge or communication information (Buckland, 1991, p.351).

Buckkand define informação como três entidades ontologicamente distintas segundo a DOLCE: como (Physical Object ->) Non-agentive Physical Object, como (Event - >) Achievement e como (Non-physical Object ->) Mental Object.

As definições de Buckland teriam também necessariamente como pressupostos atores inteligentes, sensibilizados por objetos físicos os quais desencadeiam nos mesmos processos cognitivos. Para o autor, conhecimento seria o resultado de "information-as-process". No final do mesmo texto, Buckland (1991, p.356) chega a uma conclusão desconcertante:

We conclude that we are unable to say confidently of anything that it could not be information.

This leads us to an unhelpful conclusion: If anything is, or might be, informative, then everything is, or might well be, information. In which case calling something "information" does little or nothing to define it. If everything is information, then being information is nothing special.
A visão de informação do autor integra o nível material com o mental.

Information 1 is defined as the pattern of organization of matter and energy.

Information 2 is defined as some pattern of organization of matter and energy that has been given meaning by a living being.

Knowledge is defined as information given meaning and integrated with other contents of understanding (Bates, 2005, online).

A natureza processual é clara na definição, na qual, a partir de padrões de matéria e energia, significados são extraídos por agentes/atores "vivos". A natureza de "integrated with" na definição de conhecimento é mais problemática; é um processo ("achivement"), mas não é uma mera junção de partes; seria uma integração sistêmica ao estado de conhecimento de um indivíduo ("living being"), na qual o sistema integraria a nova experiência adquirida, reorganizando-se num novo patamar de qualidade.

A visão de informação da autora integra o nível material e o mental.

Raber e Budd (2003, p.509) "Information is a kind of sign that unites text and content".

Embora outros autores em definições anteriores já falem em interpretação, num intérprete humano, essa definição se insere numa linha que vem utilizando mais explicitamente as bases teóricas da Semiótica nas questões de informação, segunda as quais um intérprete humano é um pressuposto. Estão aí explícitos artefatos "text", significado - "content" e implícito, um intérprete humano para o qual "content"faz sentido, ou seja, a definição trata um processo dito semiótico.

Capurro (2003, p.12) "A diferença entre mensagem, ou oferta de sentido, e informação, ou seleção de sentido, é, a meu ver, a diferença crucial de nossa disciplina entendida assim como teoria das mensagens e não só como teoria da informação".

Mensagens são mecanismos de comunicação intersubjetivos. Pressupõe um emissor e um receptor integrados em processos sociais concretos, ambos dotados de intencionalidade no processamento da mensagem. Embora para o receptor possa haver uma oferta, para o emissor há a intencionalidade de prover 
um sentido especifico; e para o receptor, a intenção de selecionar sentido. Assim, essa visão de informação do autor se restringe ao nível mental.

Outros autores analisados a seguir, como Frohmann (2004) e Hjørland e Albrechtsen (1995), não definem propriamente uma entidade informação. Frohmann (2004) afirma que nossas impressões abstratas do que parece ser informação são reificações de variadas práticas com documentos. Hjørland e Albrechtsen (1999) sugerem que o objeto da $\mathrm{Cl}$ deve ser conhecimento, devendo ser encontrado em domínios de conhecimento através de práticas discursivas ou de pensamento.

As definições de Frohman são as seguintes.

According to Nunberg's argument, the key properties of our abstract impression of information-as-substance, those he calls the syntactic properties of quantifiability, uniformity, and morselization (or boundedness), and the semantic properties of objectivity and autonomy, are simply the reifications of the various principles of interpretation we bring to reading specific, historically contingent document forms (Frohmann, 2004, p.388). So too does it shift our attention away from mentalistic pictures of information and toward practices with documents (p.396).

A posição do autor é esclarecida na seguinte citação:

Para mim, materialidade é o mais importante dos três principais conceitos do tema da conferência, pois muito do caráter público e social da informação depende dela. Mas, se "documento" nomeia a materialidade da informação e se sua materialidade é importante para compreender os aspectos sociais e públicos da informação, então os estudos de documentação se tornam importantes aos estudos de informação. A documentação se torna o meio de materialização da informação (Frohmann, 2006, p.2).

A visão de informação do autor se restringe ao nível social objetivado.

Por sua vez a definição de Hjørland e Albrechtsen (1995, p.400), pode ser vista na seguinte citação.

The domain analytic paradigm in Information Science (IS) states that the best way to understand information in IS is to study the knowledge-domain as thought or discourse communities [...]. The individual person's psychology, knowledge, information needs and subjective relevance criteria should be seen in this perspective.
Hjørland e Albrechtsen não definem informação em seu texto. A formulação dos autores não é, rigorosamente, uma definição do tipo $A=B$, como as tentativas anteriores, mas uma sugestão ou recomendação sobre a melhor maneira para se entender informação no contexto da Cl. No texto, não fica claro para os autores o que exatamente, num determinado domínio, seria informação. Nem se haveria alguma propriedade comum de uma entidade informação, que fosse genérica e perpassasse diferentes domínios.

Em outra formulação de Hjørland, dessa vez em conjunto com Capurro, são mencionados "objetos informativos" em relação a um domínio social: "The domain analytic view seems different objects as being informative relative to the social division of labor in society" (Capurro \& Hjørland, 2003, p.377).

Hjørland, apesar de ser um dos formuladores do chamado paradigma da "análise de domínios", no texto com Capurro, define informação como um processo cognitivo de interpretação que ocorre em nível individual, não de forma absoluta ou incondicional, mas apenas quando é processada ("informacionalmente") pelo receptor: "It may yield a message if and only if it has been processed... The message may acquire a meaning if and only if it has been information-processed by a recipient" (Capurro \& Hjørland, 2003, p.363). Isto é reafirmado em outro trecho do mesmo texto, onde a informação é então explicitamente mencionada: Information. It is a relational concept that includes the source, the signal, the release mechanism and the reaction as its relactants" (Capurro \& Hjorland, 2003, p.373).

Pode-se concluir então que a visão de informação do autor se restringe ao nível social.

\section{[...] Over the last three decades, several analysis in Information Science, in Information Systems Theory, Methodology, Analysis and Design, in Information (Systems) management, in Database Design and in Decision Theory have adopted a General Definition of Information (GDI) in terms of data + meaning (Floridi, 2013, online).}

Floridi apresenta uma definição de informação rigorosamente construída a partir do conceito de dados. O autor também delimita claramente que sua definição corresponde à informação semântica, distinguindo-a da informação na acepção de Shannon, como sequência 
de sinais numa mensagem. "Data” para Floridi se compõe de um espectro de crescente complexidade que vai desde objetos físicos, passando por perceptos, sinais, até artefatos (registros codificados, mensagens, texto), desde que sejam bem formados ("well-formed"), isto é, codificados segundo regras sintáticas de um sistema definido. A condição de bem formados sintaticamente torna dados potencialmente passíveis de se constituírem em mensagens significativas para um interprete implícito, no escopo de um processo semiótico.

Portanto, a visão de informação do autor integra o nível material ao mental.

\section{Discussão}

As categorias e relações DOLCE encontradas nas definições são apresentadas no Quadro 5.

O único termo de alguma das definições para o qual não foi identificada uma categoria DOLCE foi "Energy", encontrada na definição de Bates (2005). As categorias DOLCE para as quais nenhum dos termos das definições foi identificado são:"Abstract Region","Arbitrary Sum", "Feature", "State", "Temporal Quality" e "Temporal Region".
Exceto na formulação de Hjørland e Albrechtsen (1995), todas as outras incluem processos ("Event -> Achievement"). A única menção a entidades sociais ou coletivas ("knowledge domains"ou"discourse communities") ocorre nessa definição. Uma vez que não é uma definição rigorosa, pode-se concluir que, a menos desta e segundo as definições analisadas, informação poderia ser definida em quatro graus de complexidade, em função dos elementos envolvidos/explicitados:

a) Um processo que envolve matéria organizada, mais especificamente, objetos, naturais ou artefatos (texto, signos, documentos), que desencadeiam em atores humanos dotados de intencionalidade em sua interação com o mundo físico ou entre si (" $a$ sender", " $a$ receipt", "a living being", "someone"), alterações em seus estados mentais individuais (denotados pelo artigo "a").

A partir dessa formulação mais geral, as definições permitem identificar variações, mais restritas, no que diz respeito às entidades envolvidas, conforme se segue:

b) Um processo que envolveria objetos que desencadeiam em agentes humanos (neste caso, "a receipt", "a living being", "someone") dotados de intencionalidade em sua interação com o mundo físico ou entre si, alterações em seus estados mentais individuais. Essa

Quadro 5. Categorias e relações DOLCE usadas nas definições.

\begin{tabular}{ll}
\hline Categorias DOLCE & Termos das definições \\
\hline Event ->Achievement & $\begin{array}{l}\text { Changing, changed to by, communicated, communication, has been given, Information- } \\
\text { as-process, interpretation, Is informed, Is changed, (The act of) informing, in information-as- } \\
\text { process, imparting, oferta (oferecer), reifications, Shift away (our attention), (toward) practices } \\
\text { (with), transforming, unites. }\end{array}$ \\
$\begin{array}{ll}\text { Sender, recipient, someone, (a) living being. } \\
\text { Physical Object -> Agentive Physical Object }\end{array}$ & $\begin{array}{l}\text { Discourse communities. } \\
\text { (what they) know, structure, which is perceived, knowledge. }\end{array}$ \\
Non-physical Object -> Mental Object & mensagem, text, signs, objects. \\
Social Object -> Non-agentive Social Object & knowledge domain, thought. \\
Physical Endurant ->Amount of Matter & Matter. \\
Abstract Quality & Organization. \\
\hline Relações DOLCE & Termos das definições \\
\hline Parthood & (small) part of \\
Participation & changed to by \\
\hline
\end{tabular}

Fonte: Elaborado pelo autor (2014).

Nota: DOLCE: Descriptive Ontology for Linguistic and Cognitive Engineering. 
formulação corresponde às definições de Bates (2005) e de Buckland (1991), de "information as thing".

c) Um processo que envolve objetos específicos, artefatos (texto, signos, documentos), que desencadeiam em agentes humanos, implícitos ou explícitos, dotados de intencionalidade em sua interação com o mundo físico ou entre si ("a sender", "a receipt", "a living being", "someone"), alterações em seus estados mentais individuais. Essa formulação corresponderia às definições de Belkin e Robertson (1976), Raber e Budd (2003), Capurro (2003), Floridi (2013).

d) Um processo em que são mencionados somente alterações de estados mentais "Information is that which is capable of transforming structure", Belkin e Robertson (1976, p.198),"which is perceived in information-as-process: the knowledge communicated concerning some particular fact, subject, or event", Buckland (1991, p.351), "K [S] + $\Delta$ K $=K[S+\Delta S]$ ]", Brookes (1980a, p.131),"Knowledge is defined as information given meaning and integrated with other contents of understanding" (Bates, 2005, online).

Naturalmente nessas definições estão implícitos também atores humanos. É significativo também observar que em várias das definições os conceitos de informação e conhecimento são tomados como idênticos ou quase idênticos"I regard knowledge as a structure of concepts linked by their relations and information as a small part ofsuch astructure" (Brookes, 1980a, p.131); "Information is... the knowledge communicated concerning...", (Buckland, 1991, p.351); "The modern concept of information as knowledge communication" (Capurro \& Hjorland, 2003, p.371); "Knowledgeis defined as information given meaning and integrated with other contents of understanding" (Bates, 2005, online).

Como o objetivo deste trabalho é contribuir para uma teoria do documento e de suas relações com informação e conhecimento, procurou-se na literatura autores que propusessem definições de informação. Deve ser observado, no entanto, que vários dos autores seminais e considerados clássicos da área definem Ciência da Informação, seu escopo, mas não definem um objeto informação. Entre os autores/textos em que essa situação ocorre estão Borko (1968), Wersig e Neveling (1975), Saracevic (1992). Existe, além disso, toda uma subárea de "information retrieval", que tem como pressuposto a "informação científica", isto é, documentos científicos ou suas representações (Van Rijsbergen, 1979; Salton \& McGill, 1983; Ingwersen, 1996).

Segundo as definições, a formulação (d), um processo de alteração de estados mentais, a mais restrita e presente em todas as anteriores, constituiria então a essência do fenômeno informação nas definições analisadas. As categorias da DOLCE não permitem discriminar a mudança qualitativa dessa alteração de estados mentais, que é, segundo todas as definições, aquisição de conhecimento.

Desde o século XVII, na Crítica à Razão Pura, Kant (1991) propõe uma superação das visões racionalista e empiricista, e a Filosofia passa reconhecer a interação sujeito-objeto como necessária para a aquisição do conhecimento. Essa posição filosófica vem sendo, ao longo do tempo, corroborada por disciplinas científicas como a Epistemologia Genética, Psicologia Cognitiva e Neurociência.

Nessa linha, conhecimento é descrito por Piaget $(1983$, p.8) também como um processo: "Entretanto, sob a influência convergente de uma série de fatores, passa-se cada vez mais, hoje em dia, a considerar o conhecimento como um processo, mais que um estado". $\mathrm{O}$ autor assim descreve esse processo:

[...] não existe experiência pura no sentido do empirismo e os fatos só são acessíveis quando assimilados pelo sujeito, o que pressupõe a intervenção de instrumentos lógico-matemáticos de assimilação construtora das relações que enquadram ou estruturam esses fatos e do mesmo modo os enriquecem (Piaget, 1983, p.29)

A relação entre aquisição de conhecimento, aqui chamada de aprendizagem, memorização das experiências da vida e evocação dessas memórias para o agir do indivíduo no mundo é assim descrita, numa visão a partir das neurociências:

Desde um ponto de vista prático, a memória dos homens e dos animais é o armazenamento e evocação de informação adquirida através de experiências; a aquisição de memórias denomina-se aprendizado [...]

[...] O aprendizado e a memória são propriedades básicas do sistema nervoso; não existe atividade nervosa que não inclua ou não seja afetada de alguma forma pelo aprendizado e pela memória (Izquierdo, 1989). 
O estabelecido, na última citação de Izquierdo, de que aprendizado, memorização e rememoração são pressupostos da atividade cognitiva humana, é corroborado por Buckland (1991) quando afirma que "If anything is, or might be, informative, then everything is, or might well be, information. In which case calling something "information" does little or nothing to define it. If everything is information, then being information is nothing special". Essa questão é também enfatizada por Brookes (1980a, p.132):"I use the perceptron only to emphasise that potential information is everywhere". Assentar a $\mathrm{Cl}$ sobre bases tão amplas, genéricas e transversais (Maartens, 2007) só atrapalha a busca pelo seu estatuto científico.

O processo de aquisição de conhecimento, ou aprendizagem, como descrito acima, parece ser mais complexo do que as várias menções a processos sígnicos nos quais várias definições se apoiam e que simplesmente relacionam um signo a seu objeto e ao interpretante gerado na mente de um intérprete. Seria o que autores na área de Semiótica chamam de "juízo perceptivo" (Santaella \& Vieira, 2008, p.59): diante de uma experiência nova, tentamos, como corolário da percepção, classificá-la por semelhança a uma experiência já adquirida. Piaget $(1983$, p.9) chama esse processo de constituição de esquemas: a nova experiência e assimilada a um esquema já existente mais parecido, ou suscita a criação de um novo esquema. Conhecimento, nesse nível individual, seria assim esse processo de acúmulo de novas experiências ou "esquemas" na mente, habilitando um agir informado.

Esse processo corresponde ao que foi identificado em (a). Não se trata de um fenômeno especial ou diferente dos descritos nas citações de Piaget e Izquierdo. Além disso, como afirma esse último autor, não existe atividade cognitiva no homem que não se constitua em aprendizagem, em aquisição de conhecimento. Chamar esse processo de informação acrescenta pouco a sua compreensão, que é objeto também da Psicologia Cognitiva, da moderna Epistemologia e da Neurociência. Pode-se, no máximo, sendo específico, mas fiel ao encontrado na análise das definições, falar de um processo de informar-se, equivalente à aquisição de conhecimento ou aprendizagem.

Também deve ser observado que, segundo as citações de Piaget (1983) e Izquierdo (1989), a aquisição de conhecimento é um processo no nível individual. A aquisição de conhecimento no nível individual desde o nascimento do bebê, como ressalta Piaget, é um pressuposto para que qualquer influência social se dê. Por mais que se possa reconhecer a influência da cultura e da vida social na aquisição e organização do conhecimento (Hjørland, 2013), não há como deixar de reconhecer esse fato.

Assim como a aquisição de conhecimento é um pressuposto da atividade cognitiva do homem, assim também é um pressuposto a questão da vida em sociedade e da cultura humanas como uma segunda natureza, em paralelo à natureza biológica. Nesse contexto, a questão do conhecimento humano, sua acumulação ao longo da história humana, sua transferência entre indivíduos, primeiro através da linguagem, foi o que permitiu a interação e a articulação entre indivíduos e a vida social. Posteriormente, no contexto de sociedades cada vez mais complexas e que dependiam dessa interação e articulação para sobreviverem, à linguagem e à interação direta se adicionaram artefatos (Miller, 2005; Borgo \&Vieu, 2009) criados especificamente com essa finalidade, documentos, que permitem o registro e a transferência de mensagens através do tempo e do espaço. Esses pressupostos são dados pela história humana (Marcondes, 2010).

Em vista do que foi dito, poder-se-ia falar então de uma classificação dos processos de aquisição de conhecimento ou aprendizagem a partir de documentos ou que os contemplem. Todas as instituições sociais têm seus procedimentos baseados em documentos; todo sistema de ensino tem esta como uma de suas bases; professores, acadêmicos, educadores, estudantes em geral a praticam; manuais de metodologia científica relacionam entre seus métodos a pesquisa bibliográfica.

O que existe de específico na Clé lidar com essas extensões artificiais da memória, esses artefatos chamados registros ou documentos, cuja invenção permitiu à humanidade transferir mensagens, conhecimento, interagir e articular-se de forma indireta, não presencial, através do tempo e do espaço. Nesse contexto, interessaria à $\mathrm{Cl}$ questões como: a produção social de registros de conhecimento, sua transferência, sua acumulação e guarda, sua recuperação e utilização, enfim, a economia desses artefatos, na qual estão implícitos atores/papéis como produtor, receptor, intermediário etc. Documentos 
são socialmente instituídos, produzidos, transferidos e utilizados (Smith, 2005); não são fortuitos, ocasionais, têm uma grande objetividade dentro dos contextos sociais específicos em que são produzidos e utilizados.

Os estudos históricos em Cl (Shera, 1980), (Day, 2001) e (Burke, 2007) permitem identificar o quanto de interesses profissionais/corporativos estava envolvido no surgimento da Cl. Segundo a proposta de Le Moigne (1990, p.79), essa pesquisa complementa, do ponto de vista ontológico, aqueles estudos.

\section{Conclusão}

Como pôde ser visto, as definições oscilam entre dois polos: considerar informação como contida numa entidade material, física, ou considerar informação como fenômeno mental. As formulações da Ciência Cognitiva mostram, em se tratando da primeira acepção, que a vida consciente humana é um processo contínuo de interação com o mundo físico, de processamento das experiências de vida e aquisição de conhecimento e seu armazenamento na memória para poder evocá-las quando necessário.

Em se tratando dessa segunda acepção, esta não se distingue de processos individuais de aquisição de conhecimento, a não ser talvez pelo fato de que "informar-se" é um processo deliberado, intencional, não casual, de aquisição de conhecimento. Esse processo sempre foi, nos primórdios da humanidade, e hoje é cada vez mais, um diferencial de sucesso na sobrevivência indi- vidual ou coletiva. Defini-lo como "informação" só faz sentido como modelo explicativo, semelhante ao que faz Piaget com os esquemas, e Freud com Id, Ego e Superego, ou como é feito na Administração/Gestão do Conhecimento/Gestão da Informação; mas, mesmo nesse caso, o uso como modelo deveria ser explicitado.

Entre os polos extremos"pan-material"/"paninformativo" e mental está o processo intencional, social e sistemático de registro do conhecimento para poder viabilizar sua transferência através do espaço e do tempo, viabilizado por artefatos, objetos artificiais, documentos em seu sentido mais amplo. Esse processo permite partilhar e reusar em larga escala o conhecimento de forma econômica, sem que a humanidade precise sempre trilhar e reaprender o que já foi aprendido por alguém em algum momento. $O$ surgimento desses artefatos viabilizou que a humanidade pudesse estender, de uma forma que seria impossível somente com a linguagem, seus mecanismos de coordenação e articulação sociais, viabilizando sociedades cada vez mais complexas, nas quais o documento forma uma das bases de sustentação mais importantes. A economia dessa produção de registros/documentos, sua transferência, acumulação, distribuição e acesso são base sólida e justificativa social para $\mathrm{aCl}$.

\section{Agradecimentos}

Aos colegas professores da Faculdade de Letras da Universidade do Porto pela rica discussão e importantes contribuições.

\section{Referências}

Aristóteles. Categorias. Lisboa: Instituto Piaget, 2000.

Bates, M.J. Information and knowledge: An evolutionary frameworkfor information science. Information Research, v.10, n.4, 2005. Available from: <http://InformationR.net/ir/10-4/ paper239.html>. Cited: Jun. 14, 2013.

Belkin, N.J.; Robertson, S.E. Information science and the phenomenon of information. Journal of the American Society for Information Science, v.27, n.4, p.197-204, 1976.

Berners-Lee, T.; Hendler, J.; Lassila, O. The semantic web. Scientific American, v.284, n.5, 2001. Available from: <http:// www.scian.com/2001/0501 issue/0501 berners-lee.html>. Cited: May 18, 2013

Borgo, S.; Vieu, L. Artefacts in formal ontology. In: Meijers, A. (Ed.), Handbook of philosophy of technology and engineering sciences. Amsterdan: North-Holland, 2009. p.273-308.
Brookes, B.C. The foundations of information science: Philosophical aspects. Journal of Information Science, v.2, Part 1, p.125-133, 1980a.

Brookes, B. C. The foundations of information science: Quantitative aspects: Classes of things and the challenge of human individuality. Journal of Information Science, v.2, Part 2, p.125-133, 1980b.

Borko, H. Information science: What is it? American Documentation, v.19, n.1, p.3-5, 1968.

Buckland, M. Information as thing. Journal of the American Society of Information Science, v.42, n.5, p.351-360, 1991 Available from: <http://www.sims.berkley.edu/ buckland/ thing.html>. Cited: Apr. 13, 2009.

Burke, C. History of information science. Annual Review of Information Science and Technology, v.41, n.1, p.3-53, 2007. 
Capurro, R. Epistemologia e ciência da informação. In: Encontro Nacional de Pesquisa em Ciência da Informação, 5., 2003, Belo Horizonte. Anais eletrônicos... Belo Horizonte: Enancib, 2003. Disponível em: <http://www.capurro.de/enancib_p.htm>. Acesso em: 5 abr. 2013.

Capurro, R.; Hjorland, B. The concept of information. Annual Review of Information Science and Technology, v.37, n.8, p.343411, 2003.

Chen, P. P-S. The entity-relationship model-toward a unified view of data. ACM Transactions on Database Systems, v.1, n.1, p.9-36, 1976. Available from: <http://csc.lsu.edu/news/ erd.pdf>. Cited: Mar. 23, 2013.

Dahlberg, I. Conceptual definitions for interconcept. International Classification, v.8, n.1, p.16-22, 1981.

Dahlberg, I. Conceptual structures and systematization. International Forum on Information and Documentation, v.20, n.3, p.9-24, 1995.

Dahlberg, I. Teoria do conceito. Ciência da Informação, v.7, n.2, p.101-107, 1978. Disponível em: <http://revista.ibict.br/ciinf/ index.php/ciinf/article/viewFile/1680/1286>. Acesso em: 11 ago. 2013.

Dahlberg, I. Terminological definitions: Characteristics and demands. In: Problèmes de la définition et de la synonymie en terminologie. Québec: Girsterm, 1983. p.13-51.

Day, R.E. Modern invention of information: Discourse, history and power. Carbondale and Edwardsville: Southern Illinois University Press, 2001.

Farradane, J. Knowledge, information, and information science. Journal of Information Science, v.2, p.75-80, 1980.

Floridi, L. Semantic conceptions of information. In: Zalda, E. (Ed.).The stanford encyclopedia of philosophy, 2013. Available from: <http://plato.stanford.edu/archives/spr2013/entries/ information-semantic/>. Cited: Jan. 23, 2013.

Frohmann, B. (n.d.). Documentation Redux: Prolegomenon to (another) philosophy of information. Library Trends, v.52, n.3, p.387-407, 2004.

Frohmann, B. O caráter social, material e público da informação na contemporaneidade. In: Encontro Nacional de Pesquisa e Pós-Graduação, 7., 2006, Marília. Anais... Marília: Unesp, 2006. p.1-9.

Gnoli, C. Integrative levels classification: A general, interdisciplinary, phylogenetic, freely-faceted knowledge organization system. Milano: Isko, 2011. Available from: <http:// www.iskoi.org/ilc/book/>. Cited: Mar. 14, 2012.

Guarino, N. Formal ontology, conceptual analysis and knowledge representation. International Journal of Human Computer, v.43, p.625-640, 1995. Available from: <http:// nemo.nic.uoregon.edu/wiki/images/7/79/Guarino_I JHCS1995_Formal_Onto_conceptual_analysis.pdf>. Cited: Oct. 12, 2012.

Guarino, N. Some organizing principles for a unified top-level ontology. In: Spring Symposium on Ontological Engineering, 6., 1997, California. Proceedings electronics... California: AAAl, 1997. p.57-63. Available from: <http://www.aaai.org/Papers/ Symposia/Spring/1997/SS-97-06/SS97-0 6-008.pdf>. Cited: Jun. 14, 2013.
Hartmann, S.; Link, S. English sentence structures and EER modeling. In: Asia-Pacific Conference on Conceptual Modelling, 4., 2007, Ballarat. Proceedings electronics... Sydnei: Australian Computer Society, 2007. p.27-35. Available from: <http://dl.acm.org/citation.cfm?id=1274460>. Cited: Jul. 24, 2013.

Hjørland, B. Knowledge organization = Information organization? Advances in Knowledge Organization, v.13, 2012. Available from: <http://www.ergon-verlag.de/isko_ko/downloads/ aiko_vol_13_2012_04.pdf>. Cited: May 7, 2013.

Hjørland, B. User-based and cognitive approaches to knowledge organization. Knowledge Organizarion, v.40, n.1, p.11-27, 2013.

Hjørland, B.; Albrechtsen, H. Toward a new horizon in information science: Domain-analysis. Jasis, v.46, n.6, p.400-425, 1995

Ingwersen, P. Cognitive perspectives of information retrieval interaction: Elements of a cognitive IR theory. Journal of Documentation, v.52, n.1, p.3-50, 1996.

Izquierdo, I. Memórias. Estudos Avançados, v.3, n.6, 1989. Disponível em: <http://dx.doi.org/10.1590/S0103-401419 89000200006>. Acesso em: 21 fev. 2013.

Jacob, E.K. Ontologies and the semantic web. Bulletin of the American Society for Information Science and Technology, v.29, n.4, p.19-22, 2003.

Kant, I. Crítica à razão pura. São Paulo: Nova Cultural, 1991. (Os Pensadores, 7).

Knight, C.; Studdert-Kennedy, M.; Hurford, J.R. Language: A Darwinian adaptation? In: Knight, C.; Studdert-Kennedy, M.; Hurford, J.R. (Ed.). The evolutionary emergence of language: Social functions and the origins of linguistic form. Cambridge: Cambridge University Press, 2000. p.1-15. Available from: <http://catdir.loc.gov/catdir/samples/cam03/00020471.pdf>. Cited: Feb. 13, 2009.

Le Moigne, J.-L. A teoria do sistema geral. 3.ed. Lisboa: Instituto Piaget, 1990.

Maartens, W. The basic elements of nature: Matter, energy, and information. [S.I.]: Authorsden.com, 2007. Available from: <http://www.authorsden.com/visit/viewarticle.asp?id =30331 >. Cited: Jan. 15, 2010.

Macleod, M.C.; Rubestein, E.M. Universals. In: Internet Encyclopedia of Philosophy. 2005. Available from: <http:// www.iep.utm.edu/universa/>. Acesso em: Oct. 11, 2013.

Marcondes, C.H. Linguagem e documento: fundamentos evolutivos e culturais da ciência da informação. Perspectivas em Ciência da Informação, v.15, n.2, 2010. Disponível em: <http://portaldeperiodicos.eci.ufmg.br/index.php/pci/article/ view/1019>. Acesso em: 23 jan. 2011.

Masolo, C. et al. The wonderweb library of foundational ontologies: Preliminary report. Padova: ISTR-CNR, 2003. Available from: <http://citeseerx.ist.psu.edu/viewdoc/ download;jsessionid=F8AD1D84E4EA5C784960F1D9766 E7D99?doi=10.1.1.11.4243\&rep=rep1\&type $=p d f>$. Cited: Mar. 17, 2013. 
Miller, S. Artefacts and collective intentionality. Techné: Research in Philosophy and Technology, v.9, n.2, 2005. Available from: <http://scholar.lib.vt.edu/ejournals/SPT/v9n2/ miller.html>. Cited: Sept. 7, 2012.

Morris, C.W. Fundamentos da teoria dos signos. Rio de Janeiro: Eldourado Tijuca, 1976.

Piaget, J. A Epistemologia genética. São Paulo: Abril Cultural, 1983. (Os pensadores).

Peirce, C. S. Semiótica. São Paulo: Perspectiva, 1977.

Poli, R. The basic problem of the theory of levels of reality. Axiomathes, v.12, n.3-4, p.261-283, 2001.

Popper, K. Three worlds: The tanner lecture on human values. Michigam: University of Michigam, 1978. Available from: $<$ http://tannerlectures.utah.edu/lectures/documents/ popper80.pdf>. Cited: Apr. 14, 2013.

Raber, D.; Budd, J.M. Information as sign: Semiotics and information Science. Journal of Documentation, v.59, n.5, p.507-522, 2003. Available from: <http://www.periodicos. capes.gov.br>. Cited: Jul. 24, 2009.

Salton, G.; McGill, M.J. Introduction to modern information retrieval systems. New York: McGraw-Hil, 1983.

Santaella, L. O que é semiótica. 8.ed. São Paulo: Brasiliense, 1990. (Coleção Primeiros Passos, 103).

Santaella, L.; Vieira, J.A. Metaciência como guia da pesquisa: uma proposta semiótica e sistêmica. São Paulo: Mérito, 2008.

Saracevic, T. Information science: Origin, evolution and relations. In: Vakkari, P.; Cronin, B. (Ed.). Conceptions of Library and Information Science: Histórical, empirical and theoretical perspectives. London: Taylor Graham, 1992. p.5-27. Available from: <http://www.scils.rutgers.edu/ kantor/601/Readings 2004/Week2/w2R1.PDF>. Cited: Mar. 4, 2009.

Shera, J. Sobre biblioteconomia, documentação e ciência da informação. In: Gomes, H.E. (Ed.). Ciência da informação ou informática? Rio de Janeiro: Calunga, 1980. p.91-105.

Smith, B. Document acts. [s.I.], [2005]. Available from: <http:// ontology.buffalo.edu/document_ontology/document_ acts.doc>. Cited: Mar. 20, 2007.

Smith B. Ontology and information systems. [s.I.], [2006]. Available from: <http://ontology.buffalo.edu/ontology_ long.pdf>. Cited: May 24, 2009.

Smith, B.; Welthy, C. Ontology: Towards a new synthesis. In: International Conference on Formal Ontology in Information Systems, 2., 2001, Ogunquit. Proceedings electronics... Ongunquit: ACM Press, 2001. p.3-9. Available from: <portal. acm.org/citation.cfm?doid=505168.505201 >. Cited: May 8, 2013.

Viegas, W. Fundamentos lógicos da metodologia científica. Brasília: UnB, 2007

Van Rijsbergen, C.J. Information retrieval. Glasgow: University of Glasgow, 1979. Available from: <http://www.dcs.gla.ac.uk/ Keith/Preface.html>. Cited: May 14, 2009.

Wersig, G.; Neveling, U. The phenomena of interest to information science. The Information Scientist, v.9, n.4, p.127-140, 1975.

Zins, C. Conceptual approaches for defining data, information, and knowledge. Journal of the American Society for Information Science and Technology, v.58, n.4, p.479-493, 2007. 